\title{
Causes and outcome of rhabdomyolysis in patients admitted to medical wards in the Prince of Wales Hospital
}

\author{
Weiwei Zeng ${ }^{1 \wedge}$, Brian Tomlinson ${ }^{2,3} \wedge$ \\ ${ }^{1}$ Shenzhen Baoan Women's and Children's Hospital, Jinan University, Shenzhen, China; ${ }^{2}$ Faculty of Medicine, Macau University of Science and \\ Technology, Macau, China; ${ }^{3}$ Department of Medicine \& Therapeutics, The Chinese University of Hong Kong, Prince of Wales Hospital, Hong \\ Kong, China \\ Contributions: (I) Conception and design: B Tomlinson; (II) Administrative support: B Tomlinson; (III) Provision of study materials or patients: B \\ Tomlinson; (IV) Collection and assembly of data: W Zeng; (V) Data analysis and interpretation: W Zeng; (VI) Manuscript writing: Both authors; (VII) \\ Final approval of manuscript: Both authors. \\ Correspondence to: Brian Tomlinson, MD, FRCP. Professor, Faculty of Medicine, Macau University of Science \& Technology, Block P25, Macau \\ University of Science \& Technology, Avenida Wai Long, Taipa, Macau, China. Email: btomlinson@must.edu.mo.
}

Background: Rhabdomyolysis with a marked elevation of serum creatine kinase (CK) can be caused by various conditions. Acute kidney injury (AKI) is a potential complication of severe rhabdomyolysis and leads to a rapid increase in serum creatinine.

Methods: This study was performed to identify medical cases diagnosed with rhabdomyolysis and to examine the likely causes. Patients diagnosed with rhabdomyolysis during admission to the medical wards of Prince of Wales Hospital (PWH) in Hong Kong from January 1, 2004 to May 31, 2012 were identified by searching computer records. Details of hospital admissions were retrieved, and the underlying causes of the rhabdomyolysis and clinical outcomes were analyzed.

Results: There were 95 Chinese patients with a median age of 72 years (range, 22-92 years) assigned a diagnosis of rhabdomyolysis. A mild degree of AKI was defined as an increase of serum creatinine more than $20 \%$ above the baseline value before onset of acute illness and with the highest creatinine greater than $120 \mu \mathrm{mol} / \mathrm{L}$. Mild AKI was identified in 63 patients. Rhabdomyolysis appeared to contribute to a fatal outcome in eight patients who had multiple preexisting morbidities. The maximum CK had a median value of 9,829 U/L (range, 472-258,100 U/L). Twelve patients with peak CK levels <10× the upper limit of normal (ULN) may not have had rhabdomyolysis by this standard definition. Of the remaining 83 patients with maximum CK values $>10 \times$ the ULN, the most common contributing factors were trauma $(n=19)$ and infection ( $\mathrm{n}=17)$. Other common underlying causes included drug abuse (heroin and alcohol) and ischemia/ immobility.

Conclusions: Most patients recovered with appropriate medical interventions and had a median hospital stay of 13 days. One patient was thought to have drug-related rhabdomyolysis due to taking bezafibrate during an episode of renal impairment.

Keywords: Acute kidney injury (AKI); creatine kinase (CK); creatinine; rhabdomyolysis

Submitted Jun 22, 2021. Accepted for publication Aug 05, 2021.

doi: $10.21037 /$ atm-21-3660

View this article at: https://dx.doi.org/10.21037/atm-21-3660

^ ORCID: Weiwei Zeng, 0000-0001-6776-7087; Brian Tomlinson, 0000-0001-6717-5444. 


\section{Introduction}

The typical clinical syndrome of rhabdomyolysis is usually easy to recognize, and appropriate treatment with aggressive fluid administration and urine alkalinization is well established (1-3). However, precise diagnostic criteria for rhabdomyolysis remain elusive, and the factors which predict the progression of skeletal muscle damage are not consistent across different studies. A previous study reported that initial creatinine levels were more predictive of progression to rhabdomyolysis-associated AKI and mortality than initial creatine kinase (CK) levels (4). A recent systematic review of published articles regarding the definition of rhabdomyolysis found the most common definitions of rhabdomyolysis were plasma or serum CK levels greater than $5 \times$ the upper limit of normal (ULN) or CK levels greater than 1,000 U/L (5). The common causes of rhabdomyolysis include trauma, ischemia, infections, drugs, toxins, metabolic disorders, or an underlying genetic predisposition (2). Rhabdomyolysis can occur with many types of infection. Recently, a case of novel coronavirus disease COVID-19 presented with rhabdomyolysis involving muscle pain and weakness (6). In patients admitted to medical wards or toxicology centers, drug and chemicalrelated causes of rhabdomyolysis are of particular interest such as acute pesticide intoxication (7). Much attention has focused on the statin group of drugs, as they are very widely used and can cause muscle problems, especially with drug interactions $(8,9)$.

Rhabdomyolysis associated with statin therapy is rare, and lipid guidelines do not recommend routine monitoring of CK. However, the definition of different degrees of muscle damage by CK cutoff values is somewhat variable. Muscle symptoms with a CK level greater than $10 \times$ ULN are usually called myositis, myopathy, or severe myopathy. In contrast, rhabdomyolysis may be defined as a CK level greater than $40 \times$ ULN or sometimes greater than $50 \times$ ULN (10-12). A recent phenotypic definition for statin-related myopathy suggested rhabdomyolysis should be defined by CK elevation $>10 \times$ ULN accompanied by renal dysfunction and muscle symptoms, or CK $>50 \times$ ULN (13). However, rhabdomyolysis should be considered at lower CK values if there is severe muscular pain, general weakness, and dark urine suggesting myoglobinuria. It should also be noted that elevation of CK levels greater than $10 \times$ ULN in patients taking statins can often occur with unaccustomed physical exertion, so careful clinical assessment is necessary (12). Serum alanine transaminase (ALT) concentrations are usually elevated in rhabdomyolysis, but muscle and liver origins cannot generally be distinguished, and serum ALT levels are not usually considered to be a diagnostic criterion for rhabdomyolysis (3).

The current study was undertaken to examine a spectrum of cases with an ICD diagnosis of rhabdomyolysis who had been admitted to the medical wards of a general hospital in Hong Kong. The primary purpose was to identify the underlying causes of the rhabdomyolysis and clinical outcomes in this hospital. Secondly, we were also particularly interested in identifying cases related to statins or other drug therapies and whether there were factors that might have predicted the development of more severe muscle damage or the progression to AKI.

We present the following article in accordance with the STROBE reporting checklist (available at https://dx.doi. org/10.21037/atm-21-3660).

\section{Methods}

All subjects with a recorded diagnosis of rhabdomyolysis admitted from January 1, 2004 to May 31, 2012, to the medical wards at the Prince of Wales Hospital (PWH: a general public hospital that is the site of the Poison's Treatment Center for Hong Kong and the teaching hospital for the Faculty of Medicine of the Chinese University of Hong Kong), were included in this study. Patients were identified from the "clinical data analysis and reporting system" (CDARS) of the Clinical Management System of PWH by searching for the rhabdomyolysis diagnostic code (ICD-9 code 728.89). The patients' demographic data, disease-related information, duration and intensity of the episode, details of all drugs or herbal medicines used at the time of the episode, other medical history, diagnostic methods, management-related information, and outcome were extracted from the computer records. The authors independently assessed the causes of rhabdomyolysis and then reached a consensus by discussion. All procedures performed in this study involving human participants were in accordance with the Declaration of Helsinki (as revised in 2013). The study was approved by the Chinese University of Hong Kong-New Territories East Cluster Clinical Research Ethics Committee (registration number CRE2012.340). Individual consent for this retrospective analysis was waived.

Serum creatinine, CK, and ALT measurements were performed in the Chemical Pathology Department of PWH using the Roche Modular Analyzer (Roche Diagnostics 
Corp, Indianapolis, IN, USA) with reagents and calibrators supplied by Roche.

The definition of stage $1 \mathrm{AKI}$ according to the Kidney Disease: Improving Global Outcomes (KDIGO) guidelines is a change of $1.5-1.9 \times$ the baseline or an increase $\geq 26.5 \mu \mathrm{mol} / \mathrm{L}$ in serum creatinine (14). We identified subjects with an increase of serum creatinine more than $20 \%$ above the baseline value before onset of the acute illness and with the highest creatinine greater than the ULN for both males and females $(120 \mu \mathrm{mol} / \mathrm{L})$. This definition shows small changes in renal function, which are less than stage 1 of KDIGO.

\section{Statistical analysis}

Descriptive statistics were used to characterize subjects, etiologies, and laboratory characteristics. Percentages were used for categorical variables. Point estimates and measures of spread (mean and standard deviation) were used for continuous variables. The median and range were used to describe non-normal distributions. The concordance of CK and creatinine levels was assessed using Pearson correlation coefficients. Chi-square tests were used to assess differences in proportions, and Student's $t$-tests were used to determine if the peak CK and peak creatinine values for each of the etiologies differed from the mean values of the entire cohort. $\mathrm{P}<0.05$ was considered statistically significant.

\section{Results}

We identified 101 admissions to the PWH medical wards that were assigned the diagnostic ICD-9 code 728.89 over the study period. Two of these admissions involved readmission of patients assigned the diagnostic code in a previous admission; it appeared the code had been copied over to the subsequent admission inappropriately, so these admissions were not included in the analysis. One patient had been previously admitted to another hospital with a more severe episode of rhabdomyolysis, so this second episode was included in the analysis. Another two patients had been admitted twice, and one patient had been admitted three times with episodes of rhabdomyolysis, so altogether 99 admissions involving 95 patients were analyzed. Sixteen admissions with peak CK levels $<10 \times$ the ULN, including one patient with recurrent admissions, had been diagnosed with rhabdomyolysis by the attending doctors despite the fact this measure does not correspond with one of the standard definitions of rhabdomyolysis or severe myopathy.

Details of the 95 Chinese patients are shown in
Table 1, with subjects divided according to whether or not rhabdomyolysis was the primary diagnosis. The only significant difference $(\mathrm{P}=0.03)$ between the two groups was that there were more patients with multiple causes for rhabdomyolysis in the non-primary diagnosis group (19/63, $30 \%)$ than in the primary diagnosis group $(3 / 32,9 \%)$.

Of the 95 patients, $68 \%$ were male and, using the admission with the highest CK level in those with recurrent admissions, the median peak serum $\mathrm{CK}$ was $9,829 \mathrm{U} / \mathrm{L}$ (normal range: male 42-218; female 32-180 U/L) with a range of $472-258,100 \mathrm{U} / \mathrm{L}$ (Table 1). The median peak serum creatinine level was $183 \mu \mathrm{mol} / \mathrm{L}$ with a range of 26-1,045 $\mu \mathrm{mol} / \mathrm{L}$ (normal range: male 62-106; female $44-80 \mu \mathrm{mol} / \mathrm{L})$. The median increase in creatinine was $67.1 \%$ (range, $1.2-9,449.9 \%$ ), and the median increase in ALT was $204.8 \%$ (range, $6.7-18,021 \%$ ). Results of urine myoglobin tests were available for 61 patients, and these were positive in 32 patients (Table 1). A muscle biopsy was performed on one patient. None of the patients had a renal biopsy performed.

The primary contributing etiologies in the 83 patients with maximum CK values $>10 \times$ ULN (Table 2) included trauma $(n=19)$, infection $(n=17)$, therapeutic drugs including simvastatin or fibrates $(\mathrm{n}=9)$, heroin or alcohol abuse $(n=8)$, ischemia or immobility $(n=8)$, seizure $(n=7)$, electrolyte disorders ( $\mathrm{n}=7 ; 6$ hyponatremia, 1 hypokalemia), excessive exercise $(n=3)$, and heatstroke $(n=2)$. Neuroleptic malignant syndrome (NMS), hypothermia, and StevensJohnson syndrome (SJS; probably related to allopurinol and underlying muscle disease in a patient previously diagnosed with Duchenne muscular dystrophy) were each diagnosed in one patient, respectively. Twenty-two patients had multiple etiologies, but only the major cause is shown in Table 2 .

\section{Mortality}

Eight patients died during the hospital admission involving rhabdomyolysis (mean age 73 years, range 52-89 years). Progressive muscular disease with AKI and electrolyte disorder was a major contributing factor to the death of one patient, where the muscle biopsy showed inflammatory myopathy of an unknown cause that progressed despite treatment, including dialysis support. In four of the fatal cases, rhabdomyolysis occurred in combination with other medical problems and had either resolved by the time of death or was not considered a significant causative factor of death. In two patients, rhabdomyolysis was implicated in the development of AKI, which was a major 
Table 1 Characteristics of 95 patients with a primary or secondary diagnosis of rhabdomyolysis

\begin{tabular}{|c|c|c|c|}
\hline Variable & \multicolumn{3}{|c|}{ Cases of rhabdomyolysis } \\
\hline $\mathrm{N}$ & 95 & $32(34)$ & $63(66)$ \\
\hline Age (years) & 72 [22-92] & 73 [27-89] & 72 [22-92] \\
\hline Male, N [\%] & 65 [68] & 22 [69] & $43[68]$ \\
\hline Peak creatine kinase (U/L) & $9,829[472-258,100]$ & $8,686[1,169-126,090]$ & $10,010[472-258,100]$ \\
\hline Peak creatinine $(\mu \mathrm{mol} / \mathrm{L})$ & $183[26-1,045]$ & $153[56-1,045]$ & $194[26-1,012]$ \\
\hline Peak ALT (IU/L) & $92[12-2,480]$ & $118[24-1,120]$ & $68[12-2,480]$ \\
\hline$\%$ increase in creatinine & $67.1[1.2-944.9]$ & $80.4[1.2-781.6]$ & 67 [4-944.9] \\
\hline Days in hospital & $13[0.2-135]$ & $13.5[0.2-135]$ & $13[2-135]$ \\
\hline Patients with multiple causes, N [\%] & 22 [23] & $3[9]$ & $19[30]^{*}$ \\
\hline $\begin{array}{l}\text { Patients with }>20 \% \text { increase in creatinine, } \\
N[\%]\end{array}$ & $63[66]$ & $23[72]$ & $53[84]$ \\
\hline Urine Mb [positive/trace/negative] & $32 / 3 / 26$ & $12 / 0 / 9$ & $20 / 3 / 17$ \\
\hline Febrile, N [\%] & 28 [29] & $10[31]$ & 18 [29] \\
\hline Hypertension, $\mathrm{N}[\%]$ & $50[53]$ & $16[50]$ & $34[54]$ \\
\hline Diabetes mellitus, N [\%] & 36 [38] & 13 [41] & 23 [37] \\
\hline
\end{tabular}

Values are given as median [range] unless indicated. For patients with more than one admission, the results from the admission with the highest $\mathrm{CK}$ were chosen. ${ }^{*} \mathrm{P}<0.05$ between groups. ALT, alanine transaminase.

contributing factor to death. One of these was an 85 -yearold man who developed an ischemic leg from an embolus upon a background of dilated cardiomyopathy and atrial fibrillation. The other was an 89-year-old lady admitted with acute myocardial infarction and a history of possible injury after a fall, who developed circulatory failure. The other deceased patient was a 52-year-old man with alcohol dependence who developed rhabdomyolysis and AKI during alcohol withdrawal with excessive muscular activity and hypernatremia. He died from acute subarachnoid hemorrhage that may have been precipitated by some of these events.

\section{Patients with possible drug-related rhabdomyolysis}

Nine patients taking statins $(n=7)$ or fibrates $(n=2)$ at the time of the rhabdomyolysis episode were grouped as possible drug-related rhabdomyolysis. However, a review of these cases suggested that in patients taking statins, the drug did not contribute to the onset of rhabdomyolysis. Most cases had multiple contributing factors. One patient was a 50-year-old lady with diabetic nephropathy, hypertension, and obesity who was admitted with urinary tract infection and developed AKI with plasma creatinine rising from 327 to $651 \mu \mathrm{mol} / \mathrm{L}$. Four days later, her CK level peaked at $39,250 \mathrm{U} / \mathrm{L}$. She was taking bezafibrate $400 \mathrm{mg}$ daily and ezetimibe $10 \mathrm{mg}$ daily, and it is likely that with the worsening of her renal function the bezafibrate resulted in muscle toxicity. Both drugs were withdrawn, and the patient recovered. This patient had previously developed asymptomatic myositis (CK 2,414 U/L) when taking atorvastatin $20 \mathrm{mg}$ and developed a mild elevation of CK 
Table 2 Main etiological factors in 83 patients with rhabdomyolysis and maximum creatine kinase $>10$ times the ULN

\begin{tabular}{|c|c|c|c|c|c|c|c|c|}
\hline Category & No. [\%] & $\begin{array}{l}\text { Peak creatinine } \\
(\mu \mathrm{mol} / \mathrm{L})\end{array}$ & Peak CK (U/L) & Peak ALT (U/L) & $\begin{array}{l}\text { Days in } \\
\text { hospital }\end{array}$ & AKI & $\begin{array}{l}\text { Multiple } \\
\text { causes }\end{array}$ & Deaths \\
\hline Trauma & 19 [23] & $188.3 \pm 158.8$ & $20,816 \pm 27,073$ & $151.3 \pm 209.5$ & $24 \pm 17$ & 11 & 5 & 1 \\
\hline Statin/fibrate & $9[11]$ & $262.8 \pm 206.0$ & $37,775 \pm 61,262$ & $212.9 \pm 165.6$ & $16 \pm 18$ & 7 & 1 & 0 \\
\hline Electrolyte disorder & 7 [8] & $175.0 \pm 159.0$ & $13,020 \pm 11,412$ & $97.6 \pm 112.6$ & $15 \pm 13$ & 4 & 3 & 1 \\
\hline Seizure & $6[7]$ & $124.5 \pm 77.0^{*}$ & $46,395 \pm 61,621$ & $215.5 \pm 278.6$ & $14 \pm 11$ & 3 & 3 & 0 \\
\hline Exercise & $3[4]$ & $82.0 \pm 10.4^{\star \star}$ & $68,665 \pm 54,612$ & $252.7 \pm 283.8$ & $19 \pm 24$ & 2 & 0 & 0 \\
\hline SJS & $1[1]$ & 293.0 & 9,829 & NA & 135 & 1 & 1 & 0 \\
\hline Muscle disease & $1[1]$ & $<20$ & 3,064 & NA & 34 & 0 & 0 & 0 \\
\hline
\end{tabular}

Values are mean $\pm \mathrm{SD}$ or number [\%]. ${ }^{*} \mathrm{P}<0.05,{ }^{* \star} \mathrm{P}<0.01$ compared to all 83 patients. ULN, upper limit of normal; CK, creatine kinase; ALT, alanine transaminase; AKI, acute kidney injury; NMS, neuroleptic malignant syndrome; SJS, Stevens-Johnson syndrome.

(332 U/L) when taking simvastatin $10 \mathrm{mg}$, so she may have had some underlying muscle disease that predisposed her to drug-induced muscle toxicity.

Another patient taking gemfibrozil suffered from immobility after a fall, and it was not clear whether the gemfibrozil contributed to the subsequent rhabdomyolysis and AKI. At the time of rhabdomyolysis onset, seven patients were taking simvastatin, which was the most commonly prescribed statin at that time. They all had multiple other medical problems that likely contributed to rhabdomyolysis. The dose of simvastatin had not been changed recently, and there had been no recent addition of other drugs likely to interact with simvastatin or other factors that might predispose to simvastatin toxicity. Therefore, we concluded that simvastatin had not contributed significantly to the rhabdomyolysis in these cases.

\section{Acute kidney injury (AKI)}

We identified subjects with a small decline in renal function, defined as an increase of serum creatinine more than $20 \%$ above the baseline value before onset of acute illness and with the highest creatinine greater than $120 \mu \mathrm{mol} / \mathrm{L}$. The number and percentage of patients with stage 1,2 , and 3
AKI as defined by KDIGO in 2011 are shown in Table 1. Four patients who had preexisting chronic renal failure were excluded from the analysis. There were $63(66 \%)$ patients who developed AKI by our definition. The peak creatinine level was statistically lower in cases caused by seizure compared to the entire cohort (Table 2). None of the etiological categories appeared to be associated with an increased risk of AKI.

The peak CK was not significantly different between groups with differing etiological factors or for each etiological category compared to the entire cohort. In contrast, peak ALT was statistically lower in cases caused by infection and statistically higher in cases caused by exercise compared with the entire cohort. The percentage increase in serum creatinine showed a weak but significant correlation with peak $C K$ values (linear regression $R^{2}=0.15$, $\mathrm{P}<0.001)$.

\section{Duration of hospital stay}

For the total number of 95 patients, the median hospital stay was 13 days, which included the eight patients who died and one patient who discharged himself against medical advice. The development of more than a $20 \%$ increase in creatinine was associated with a significantly $(\mathrm{P}=0.001)$ 
longer hospital stay (median 21 days, range 2-135 days) compared with those without this increase in creatinine (median 8 days, range 2-60 days). There were no significant differences in the duration of hospital stay between each etiological category compared to the entire cohort.

\section{Discussion}

The main limitation of this study is that it was based on the diagnosis of rhabdomyolysis being made and entered into the electronic records system by the doctors attending the patients. The diagnosis may have been made by clinical judgment rather than relying on a specific elevation in CK levels or the presence of myoglobinuria, and some of the patients given a diagnosis of rhabdomyolysis did not have AKI or a marked elevation in CK. This approach may have resulted in many true cases of rhabdomyolysis being missed and some cases being given the diagnosis inappropriately. Regarding the 16 patients with peak CK levels $<10 \times$ the ULN, the diagnosis may have been assigned because of the presence of AKI that may have preceded or coincided with a small increase in CK, but from retrospective examination of these cases the two phenomena may not have been related, and the diagnosis may not have been appropriate.

Similar to previous reports, the most common etiological factors for rhabdomyolysis in this study of medical ward admissions were trauma and infection (4). The next most common causes were heroin or alcohol abuse, ischemia or immobility, seizure, and electrolyte disorders. Multiple causes were present in $22(23 \%)$ patients.

In one of the largest reported studies of 475 patients with acute neuromuscular illness and serum CK more than $5 \times$ ULN from the Johns Hopkins Hospital inpatient records, exogenous toxins were the most common cause of rhabdomyolysis, with illicit drugs, alcohol, and prescribed drugs accounting for $46 \%$ of cases. Multiple etiological factors were present in $60 \%$ of all cases (15). Numerous etiological factors were also related to the development of AKI.

Another retrospective review of 97 patients attending an emergency department in the United States found the most common causes of rhabdomyolysis were cocaine (30 cases), exercise (29 cases), and immobilization (18 cases). In 17 patients who developed AKI, the only variable predictive of both AKI and the need for hemodialysis was the initial serum creatinine level (16). Illicit drug use as a cause of rhabdomyolysis was associated with AKI in another recent study from Melbourne (17).
Vigorous unaccustomed exercise can result in severe rhabdomyolysis and AKI $(18,19)$. Exercise-related rhabdomyolysis occurred in three patients in our study, with two developing AKI. In rare cases of severe or recurrent episodes of exertional rhabdomyolysis, this may be a manifestation of an underlying muscle disorder, and it is important to identify factors that predict the risk of increased morbidity and mortality (20). In a study of subjects enrolled in basic military training, exertional rhabdomyolysis was very uncommon with minimal risk of recurrence, and it was less likely to result in renal failure than other causes of rhabdomyolysis (21). The initial presentation of exertional rhabdomyolysis may be subtle, so it is important to consider this before AKI develops (22).

The proposed mechanisms for the development of rhabdomyolysis induced by many types of infection include tissue hypoxia secondary to sepsis or dehydration, toxin release, associated fever, direct bacterial invasion of muscle, or rigors/tremors (23). Muscle toxicity related to statin therapy has received much attention in recent years $(8,12,24)$. It has been suggested that warning patients of potential muscle problems may result in the nocebo effect, where muscle pain is attributed to the statin therapy thus creating apparent statin intolerance $(25,26)$. The only patient in this case series where rhabdomyolysis may have been prevented or reduced was the patient with diabetic nephropathy and possible underlying muscle disease who was taking bezafibrate during a period of AKI. Toxicity from simvastatin was not the obvious primary cause of rhabdomyolysis in the seven patients taking that drug.

A recent case of simvastatin-related muscle toxicity reported from Hong Kong involved a patient taking simvastatin $40 \mathrm{mg}$ daily for over 10 years who developed acute rhabdomyolysis, possibly related to a mild drug interaction with a newly started drug or herbal medicine (27). Genetic analysis revealed that the patient carried one copy of the c.521T>C (rs4149056) variant in the gene encoding the organic anion-transporting polypeptide 1B1 (OATP1B1, gene SLCO1B1). It is recommended that patients with this genotype should not be given doses of simvastatin as high as $40 \mathrm{mg}$ due to the risk of severe myopathy (28). Indeed, Chinese patients demonstrate higher plasma concentrations than Caucasians when prescribed most statins, and it has been recommended that the dosage of statins should be reduced in Chinese patients. This is a similar recommendation to the maximum approved doses in Japan, which are lower than in Western countries (29). 
In the present study, the duration of hospital stay was longer for those patients who developed AKI, so early aggressive treatment of suspected rhabdomyolysis may help to prevent the development or progression of AKI and hasten recovery. We did not identify any obvious etiological factors associated with the development of AKI, and we could not distinguish between maximum levels of CK or creatinine as being more predictive of developing AKI.

Of the patients that died, rhabdomyolysis was not the main cause of death except for the patient with inflammatory myopathy of unknown cause. A study found in critically ill patients with rhabdomyolysis, patients who had a ALT over 1,000 U/L had a higher mortality than those with levels below 1,000 U/L (61\% vs. 15\%) (30).

Overall, it is unlikely that more aggressive treatment of the rhabdomyolysis or AKI would have altered the outcome in the other cases. We did not identify the need for any improvement in the management of these patients, except that fibrate treatment should be stopped earlier when renal function deteriorates. The main principles of management for rhabdomyolysis are treatment with aggressive fluid administration and urine alkalinization, although the clinical benefits of alkalinization as compared with simple volume repletion are not firmly established. Any obvious underlying causes, such as infection, should be treated appropriately and if hyperkalemia occurs this should be managed carefully and renal-replacement therapy may be required in severe cases of AKI (31).

\section{Conclusions}

Of the 95 patients admitted to medical wards over this period who received a diagnosis of rhabdomyolysis, renal impairment of a mild degree or greater occurred in 63 patients. The common underlying causes of rhabdomyolysis were trauma, infection, drug abuse, and ischemia/immobility. None of these underlying causes were associated with an increased risk of developing AKI or had a major influence on the duration of hospital stay. Some patients may have received a diagnosis of rhabdomyolysis due to the presence of AKI with a slight increase in CK.

In the nine patients prescribed either statins or fibrates at the time of onset of rhabdomyolysis, the drug was probably only directly related to rhabdomyolysis in one case who was prescribed bezafibrate. Most patients recovered with appropriate medical interventions, and for those patients who died, their deaths were mainly caused by multiple comorbidities.

\section{Acknowledgments}

Funding: None.

\section{Footnote}

Reporting Checklist: The authors have completed the STROBE reporting checklist. Available at https://dx.doi. org/10.21037/atm-21-3660

Data Sharing Statement: Available at https://dx.doi. org/10.21037/atm-21-3660

Conflicts of Interest: Both authors have completed the ICMJE uniform disclosure form (available at https://dx.doi. org/10.21037/atm-21-3660). The authors have no conflicts of interest to declare.

Ethical Statement: The authors are accountable for all aspects of the work in ensuring that questions related to the accuracy or integrity of any part of the work are appropriately investigated and resolved. All procedures performed in this study involving human participants were in accordance with the Declaration of Helsinki (as revised in 2013). The study was approved by the Chinese University of Hong Kong - New Territories East Cluster Clinical Research Ethics Committee (registration number CRE-2012.340). Individual consent for this retrospective analysis was waived.

Open Access Statement: This is an Open Access article distributed in accordance with the Creative Commons Attribution-NonCommercial-NoDerivs 4.0 International License (CC BY-NC-ND 4.0), which permits the noncommercial replication and distribution of the article with the strict proviso that no changes or edits are made and the original work is properly cited (including links to both the formal publication through the relevant DOI and the license). See: https://creativecommons.org/licenses/by-nc-nd/4.0/.

\section{References}

1. Chatzizisis YS, Misirli G, Hatzitolios AI, et al. The syndrome of rhabdomyolysis: complications and treatment. Eur J Intern Med 2008;19:568-74.

2. Giannoglou GD, Chatzizisis YS, Misirli G. The syndrome of rhabdomyolysis: Pathophysiology and diagnosis. Eur J Intern Med 2007;18:90-100.

3. Bosch X, Poch E, Grau JM. Rhabdomyolysis and acute 
kidney injury. N Engl J Med 2009;361:62-72.

4. Baeza-Trinidad R, Brea-Hernando A, Morera-Rodriguez $\mathrm{S}$, et al. Creatinine as predictor value of mortality and acute kidney injury in rhabdomyolysis. Intern Med J 2015;45:1173-8.

5. Stahl K, Rastelli E, Schoser B. A systematic review on the definition of rhabdomyolysis. J Neurol 2020;267:877-82.

6. Suwanwongse K, Shabarek N. Rhabdomyolysis as a Presentation of 2019 Novel Coronavirus Disease. Cureus 2020;12:e7561.

7. Park JS, Seo MS, Gil HW, et al. Incidence, etiology, and outcomes of rhabdomyolysis in a single tertiary referral center. J Korean Med Sci 2013;28:1194-9.

8. Magni P, Macchi C, Morlotti B, et al. Risk identification and possible countermeasures for muscle adverse effects during statin therapy. Eur J Intern Med 2015;26:82-8.

9. Page SR, Yee KC. Rhabdomyolysis in association with simvastatin and dosage increment in clarithromycin. Intern Med J 2014;44:690-3.

10. Turner RM, Pirmohamed M. Statin-Related Myotoxicity: A Comprehensive Review of Pharmacokinetic, Pharmacogenomic and Muscle Components. J Clin Med 2019;9:22.

11. Newman CB, Preiss D, Tobert JA, et al. Statin Safety and Associated Adverse Events: A Scientific Statement From the American Heart Association. Arterioscler Thromb Vasc Biol 2019;39:e38-81.

12. Stroes ES, Thompson PD, Corsini A, et al. Statinassociated muscle symptoms: impact on statin therapyEuropean Atherosclerosis Society Consensus Panel Statement on Assessment, Aetiology and Management. Eur Heart J 2015;36:1012-22.

13. Alfirevic A, Neely D, Armitage J, et al. Phenotype standardization for statin-induced myotoxicity. Clin Pharmacol Ther 2014;96:470-6.

14. Section 2: AKI Definition. Kidney Int Suppl 2012;2:19-36.

15. Melli G, Chaudhry V, Cornblath DR. Rhabdomyolysis: an evaluation of 475 hospitalized patients. Medicine (Baltimore) 2005;84:377-85.

16. Fernandez WG, Hung O, Bruno GR, et al. Factors predictive of acute renal failure and need for hemodialysis among ED patients with rhabdomyolysis. Am J Emerg Med 2005;23:1-7.

17. Lau Hing Yim C, Wong EWW, Jellie LJ, et al. Illicit drug use and acute kidney injury in patients admitted to hospital with rhabdomyolysis. Intern Med J 2019;49:1285-92.

18. Peng F, Lin X, Sun LZ, et al. Exertional rhabdomyolysis in a 21 -year-old healthy man resulting from lower extremity training: A case report. Medicine (Baltimore) 2019;98:e16244.

19. McVane BA, Andreae MC, Fernando DB, et al. Exertional Rhabdomyolysis in a Long-Distance Migrant. J Emerg Med 2019;56:551-3.

20. Heytens K, De Ridder W, De Bleecker J, et al. Exertional rhabdomyolysis: Relevance of clinical and laboratory findings, and clues for investigation. Anaesth Intensive Care 2019;47:128-33.

21. Alpers JP, Jones LK Jr. Natural history of exertional rhabdomyolysis: a population-based analysis. Muscle Nerve 2010;42:487-91.

22. Furman J. When exercise causes exertional rhabdomyolysis. JAAPA 2015;28:38-43.

23. Singh U, Scheld WM. Infectious etiologies of rhabdomyolysis: three case reports and review. Clin Infect Dis 1996;22:642-9.

24. Sathasivam S. Statin induced myotoxicity. Eur J Intern Med 2012;23:317-24.

25. Collins R, Reith C, Emberson J, et al. Interpretation of the evidence for the efficacy and safety of statin therapy. Lancet 2016;388:2532-61.

26. Peto R, Collins R. Trust the Blinded Randomized Evidence That Statin Therapy Rarely Causes Symptomatic Side Effects. Circulation 2018;138:1499-501.

27. Chan JCM, Ng MH, Wong RSM, et al. A case of simvastatin-induced myopathy with SLCO1B1 genetic predisposition and co-ingestion of linagliptin and Stevia rebaudiana. J Clin Pharm Ther 2019;44:381-3.

28. Wilke RA, Ramsey LB, Johnson SG, et al. The clinical pharmacogenomics implementation consortium: CPIC guideline for SLCO1B1 and simvastatin-induced myopathy. Clin Pharmacol Ther 2012;92:112-7.

29. Tomlinson B, Chan P, Liu ZM. Statin Intolerance-An Asian Perspective. J Atheroscler Thromb 2020;27:485-8.

30. Raurich JM, Llompart-Pou JA, Rodríguez-Yago M, et al. Role of Elevated Aminotransferases in ICU Patients with Rhabdomyolysis. Am Surg 2015;81:1209-15.

31. Bosch X, Poch E, Grau JM. Rhabdomyolysis and acute kidney injury. N Engl J Med 2009;361:62-72.

(English Language Editor: D. Fitzgerald)

Cite this article as: Zeng W, Tomlinson B. Causes and outcome of rhabdomyolysis in patients admitted to medical wards in the Prince of Wales Hospital. Ann Transl Med 2021;9(16):1329. doi: 10.21037/atm-21-3660 